\title{
Impact of the Covid-19 pandemic on rates of emergency department utilization and hospital admission due to general surgery conditions
}

\author{
Saba Balvardi ${ }^{1,2} \cdot$ Josie Cipolla $^{1} \cdot$ Nawar Touma $^{2} \cdot$ Tharaniya Vallipuram $^{2} \cdot$ Natasha Barone $^{2} \cdot$ Reginold Sivarajan $^{2}$. \\ Pepa Kaneva $^{2} \cdot$ Sebastian Demyttenaere $^{1} \cdot$ Marylise Boutros $^{1} \cdot$ Lawrence Lee $^{1,2} \cdot$ Liane S. Feldman $^{1,2}$. \\ Julio F. Fiore $\mathrm{Jr}^{1,2,3}$
}

Received: 30 August 2021 / Accepted: 9 December 2021 / Published online: 3 January 2022

(c) The Author(s), under exclusive licence to Springer Science+Business Media, LLC, part of Springer Nature 2021

\begin{abstract}
Background Recent literature reports a decrease in healthcare-seeking behaviours by adults during the Covid-19 pandemic. Given that emergency general surgery (GS) conditions are often associated with high morbidity and mortality if left untreated, the objective of this study was to describe and quantify the impact of the Covid-19 pandemic on rates of emergency department (ED) utilization and hospital admission due to GS conditions.

Methods This cohort study involved the analysis of an institutional database and retrospective chart review. We identified adult patients presenting to the ED in a network of three teaching hospitals in Montreal, Canada during the first wave of the Covid-19 pandemic (March13-May13, 2020) and a control pre-pandemic period (March13-May13, 2019). Patients with GS conditions were included in the analysis. ED utilization rates, admission rates and 30-day outcomes were compared between the two periods using multivariate regression analysis.

Results During the pandemic period, 258 patients presented to ED with a GS diagnosis compared to 351 patients pre-pandemically (adjusted rate ratio (aRR) $0.75 ; p<0.001$ ). Rate of hospital admission during the pandemic was also significantly lower $(\mathrm{aRR}=0.77, p<0.001)$. Patients had a significantly shorter ED stay during the pandemic (adjusted mean difference $5.0 \mathrm{~h} ; p<0.001$ ). Rates of operative management during the pandemic were preserved compared to the pre-pandemic period. There were no differences in 30-day complications (adjusted odds ratio (aOR) 1.46; $p=0.07$ ), ED revisits (aOR 1.10; $p=0.66$ ) and (re)admissions (aOR 1.42; $p=0.22$ ) between the two periods.

Conclusion There was a decrease in rates of ED utilization and hospital admissions due to GS conditions during the first wave of the Covid -19 pandemic; however, rates of operative management, complications and healthcare reutilization were unchanged. Although our findings are not generalizable to patients who did not seek healthcare, it was possible to successfully uphold institutional standards of care once patients presented to the ED.
\end{abstract}

Keywords Covid-19 $\cdot$ General surgery conditions $\cdot$ Surgical outcomes $\cdot$ Emergency room utilization

In December 2019, a novel coronavirus (Covid-19) was identified as the causative agent of an outbreak of pneumonia [1]. In the coming months, the virus had spread

Julio F. Fiore Jr

julio.fiorejunior@mcgill.ca

1 Department of Surgery, McGill University, Montreal, QC, Canada

2 Steinberg-Bernstein Centre for Minimally Invasive Surgery and Innovation, McGill University Health Centre, Montreal, QC, Canada

3 Montreal General Hospital, 1650 Cedar Ave, R2-104, Montreal, QC H3G 1A4, Canada world-wide to cause a major global pandemic [1]. The first case of Covid -19 in Quebec, Canada was diagnosed on February 28th, 2020 [2]. As a response, in line with the World Health Organization recommendations [3], the Quebec government implemented a province-wide order of lockdown and self-isolation on March 13, 2020 which lasted until the end of June of 2020 (first pandemic wave in Quebec) [2]. To expand the healthcare system capacity to treat patients with Covid-19, the Quebec Ministry of Health and Social Services employed a comprehensive strategy which included the repurposing of surgical care infrastructure and delaying non-essential surgical procedures [4]. In other words, hospitals across the province had to limit surgeries to only 
urgent cases, including those presenting to the emergency department (ED) requiring prompt intervention.

Recent literature supports that patients' fear of being infected by Covid-19 [5] and concerns about oversaturation of EDs have led to an alarming decrease in emergency careseeking behaviours around the world [1, 6-8]. As a result, recent studies have reported an increase in morbidity rates [9] and, in countries such as Italy, there has been a significant increase in mortality which cannot be fully explained by Covid-19 cases alone [10]. Since the beginning of the pandemic, significant efforts have been devoted to developing a better understanding of the Covid-19 disease process, prevention, and treatment. However, there has been limited focus on the collateral damage of this pandemic on patients requiring care for other serious illnesses.

Emergency general surgery conditions such as hollow viscus perforation, appendicitis, acute cholecystitis, and diverticulitis represent a unique group of acute illnesses with high risk of morbidity and mortality if left untreated [11, 12]. Therefore, delayed seeking of emergency care by patients suffering from these conditions may lead to debilitating and life-threatening disease progression. A better understanding of changes in patterns of ED presentation of patients with acute general surgery conditions can shed light on the collateral healthcare damage of the Covid-19 pandemic and inform the organization of emergency surgery capacity going forward. Therefore, the aim of this study was to assess the extent to which the COVID-19 pandemic impacted rates of emergency department (ED) utilization and hospital admissions due to general surgery conditions in comparison to a control pre-pandemic period.

\section{Materials and methods}

\section{Study design and participants}

This cohort study involved the analysis of an institutional database (MUHC Data Warehouse) supplemented by retrospective chart review. The study was approved by our institutional research ethics board (ref. MUHC REB MP-372021-6721). Analysis and reporting were in accordance with the STROBE Statement for cohort studies (Supplementary Material) [13]. The institutional database was used to identify and retrieve all the ED visits of patients with general surgical conditions presenting to adult hospitals that are part of the McGill University Health Centre (MUHC).

Adult ( $\geq 18$ years) patients who visited the ED during the first two months after the implementation of the government mandated lockdown were identified. This period was defined as March 13-May 13, 2020 (pandemic period) and the corresponding period the year before was defined as the pre-pandemic baseline (March 13-May 13, 2019).
Those with a confirmed diagnosis of a general surgical condition, based on the American Association for the Surgery of Trauma (AAST) compiled list of emergency general surgery pathologies [14] were included. As per AAST, trauma and post-transplant patients were excluded. Cardiovascular and plastics surgery emergencies that are not managed by general surgeons in Canadian tertiary care centres were also excluded.

\section{Outcomes}

The primary outcomes for this study were a priori defined as:

The number of patients presenting the ED with general surgery conditions.

The number of hospital admissions of patients presenting to the ED with general surgery conditions.

Secondary a priori outcomes included:

ED length of stay, defined as the number of hours lapsed between triage and discharge from the ED or admission to an inpatient care unit;

ED wait times, defined as three different entities: time to be seen by a medical doctor (MD), time to completion of consults, and time to admission from the ED. These time periods were calculated in hours from the time of arrival triage;

Hospital length of stay, defined as the number of days lapsed between admission to an inpatient unit and hospital discharge (or death);

Duration of symptoms prior to ED presentation, defined as the number of days of symptoms (of the chief complaint) prior to presenting to ED;

30-day complications, defined as any medical or surgical complications up to 30 days after the index presentation to ED [15];

30-day ED revisits, defined as any visit to the ED up to 30 days after the index presentation to the ED;

30-day (re)admission, defined as any non-elective medical or surgical admission or readmission to the hospital 30 days after the index presentation to the ED;

\section{Data collection and follow-up procedures}

Data regarding the ED episode of care (i.e. time of triage, triage categories, mode of arrival, time of first encounter by $\mathrm{MD}$, time of general surgery consult fulfillment, final diagnosis, time, and type of admission) were available through our institutional database. Triage categories used at the MUHC are based on the Canadian triage and acuity scale [16]. Patient demographics (i.e. age, sex, body mass 
index, medical comorbidities, psychiatric comorbidities, average household income) and patient outcome data (i.e. complications, ED revisits, (re)admissions) were obtained from retrospective review of electronic medical records. Medical comorbidities were classified based on the Charlson Comorbidity Index (CCI) and categorized into patients with high comorbidity $(\mathrm{CCI}>2)$ versus low comorbidity $(\mathrm{CCI} \leq 2)[17]$. Psychiatric comorbidities were recorded [18] and presented in two categories (no psychiatric comorbidity versus presence of any psychiatric comorbidity). Patient income (average household income) was used as a surrogate measure for patient socioeconomic status. This measure was calculated based on the patients' postal code which was accessible through a publicly available online tool (PRIZM ${ }^{\circledR}$ Premier) developed by Environics Analytics (https://prizm. environicsanalytics.com). Patients were divided into groups, based on income quantiles. Across the MUHC hospitals, Covid-19 testing (polymerase chain reaction technique) was not universally done for all the ED visits during the first wave of the pandemic. Test results from patients presenting with potential COVID-related symptoms were collected when available.

General surgery diagnoses were grouped into the following categories: anorectal disease, appendicitis, bowel obstruction, cancer diagnosis (primary diagnosis), cholangitis, diverticulitis, Gallstone diseases, gastrointestinal bleed, hollow viscus perforation, soft tissue infection, allcause pancreatitis, and postoperative complications. Bowel obstruction diagnosis consisted of small and large bowel obstruction regardless of cause. Gallstone diseases were defined as patients with biliary colic, acute cholecystitis, choledocholithiasis and stone cholangitis. Cholangitis category included all non-stone causes of cholangitis (i.e. post instrumentalization and stenting, or obstructing mass). Soft tissue infection consisted of severe cellulitis, abscess, and necrotizing fasciitis.

\section{Statistical analysis}

The sample size for this study was based on the number of eligible patients presenting to the ED during the study periods; as such, no formal sample size calculation was conducted. Continuous variables were summarized using mean and standard deviation or median and interquartile range, as appropriate. Categorical variables were summarized using frequencies and percentages. Chi-square and Student's $t$ tests were used for univariate analyses of categorical and normally distributed continuous variables, respectively. Poisson exact test was used for univariate comparison of count data (number of patients presenting to ED with general surgery condition, number of hospital admissions and number of patients with each diagnostic category).
To improve precision and address potential betweengroup differences in patient characteristics, multivariate regression analysis was used for comparison of a priori defined primary and secondary outcomes. Poisson regression was used for analysis of count estimates (ED visits with general surgery diagnosis and hospital admissions with general surgery diagnosis). Logistic regression was used for analysis of binary outcomes (30-day complication, 30-day ED revisits and 30-day readmissions). Linear regression was used for analyzing continuous outcomes (ED length of stay, hospital length of stay and duration of symptoms prior to presentation). Estimates were adjusted for sex, age, CCI category, psychiatric comorbidities, and income quantiles [17, 19-24]. All statistical tests were 2-sided and statistical significance was set at $p<0.05$. We conducted a complete case analysis (with no imputation of missing data) as there were no missing data for primary outcome measures and the rate of missing data for secondary outcomes and covariates were less than 5\% (Supplementary Material) [25]. Statistical analyses were performed using RStudio (version 1.2.1577; RStudio, Inc., Boston, MA, USA).

\section{Results}

During the pre-pandemic period (March13-May13, 2019), 351 patients presented to the ED with a confirmed general surgery diagnosis, compared to 258 patients during the pandemic period (March13-May 13, 2020) $(p<0.001)$. This represents a $27 \%$ decrease in the number of patients presenting to the ED with general surgery conditions in comparison to the pre-pandemic baseline. Patient demographics were similar between the two time periods, including age (55.2 \pm 19.6 years [2019] versus $55.3 \pm 19.6$ [2020]), sex (53.0\% male [2019] versus $52.0 \%$ male [2020]) and CCI (57.0\% with low comorbidity [2019] versus $58.5 \%$ low comorbidity [2020]) (Table 1).

Emergency department care characteristics for these patients are described in Table 2. According to the Canadian triage and acuity scale, there were 128 [2019] versus 77 [2020] patients presenting to the ED with emergent care needs (60.1\% of baseline [2019]). Duration of symptoms prior to presentation to the ED was not significantly different between the two time periods $(3.7 \pm 6.2$ days [2019] versus $3.7 \pm 5.6$ days [2020], $p=0.98$ ). Length of ED stay (25.2 \pm 20.7 days [2019] versus $20.1 \pm 16.0$ days [2020], $p<0.001)$ and time to admission $(14.6 \pm 11.9$ days [2019] versus $12.4 \pm 8.4$ days [2020], $p<0.001$ ) were significantly shorter during the pandemic period.

The distribution of general surgery diagnoses in the ED during the pandemic and pre-pandemic periods is described in Fig. 1. Presentations due to anorectal disease, appendicitis, bowel obstruction, cancer diagnosis, cholangitis, 
Table 1 Patient demographics during the pandemic (March13May13, 2020) and prepandemic (March13-May13, 2019) periods

\begin{tabular}{llll}
\hline & 2019 & 2020 & $p$-value \\
\hline Age, years & $55.2 \pm 19.6$ & $55.3 \pm 19.6$ & 0.96 \\
Sex (Male), $n(\%)$ & $183(53.0 \%)$ & $135(52.0 \%)$ & 0.96 \\
BMI, Kg/m & $34.1 \pm 56.7$ & $29.1 \pm 24.0$ & 0.33 \\
Medical comorbidities & & & \\
Charlson Comorbidity Index ( $\leq 2), \mathrm{n}(\%)$ & $200(57.0 \%)$ & $151(58.5 \%)$ & 0.70 \\
Psychiatric comorbidities, $n(\%)$ & $42(12.0 \%)$ & $20(7.8 \%)$ & 0.09 \\
Income, CAD & $85,368(51,637)$ & $85,368(53,132)$ & 0.24 \\
1st Income Quantile, $n(\%)$ & $71(20.2 \%)$ & $62(24.0 \%)$ & \\
2nd Income Quantile, $n(\%)$ & $89(25.3 \%)$ & $81(31.4 \%)$ & \\
3rd Income Quantile, $n(\%)$ & $88(25.1 \%)$ & $56(21.7 \%)$ & \\
4th Income Quantile, $n(\%)$ & $92(26.2 \%)$ & $55(21.7 \%)$ & \\
Total, n & 351 & 258 & \\
\hline
\end{tabular}

Data are presented as $n$ (\% total), median (IQR), or mean \pm standard deviation

$B M I$ Body Mass Index, $C A D$ Canadian Dollars

\begin{tabular}{llll}
\hline & 2019 & 2020 & $p$-value \\
\hline $\begin{array}{lll}\text { Patients with general surgery diagnosis, } n \\
\text { Canadian triage and acuity scale, } n(\%)\end{array}$ & 351 & 258 & $<\mathbf{0 . 0 0 1}$ \\
Level 1: Resuscitation & 0 & 0 & 0.19 \\
Level 2: Emergent & $128(36.5 \%)$ & $77(29.8 \%)$ & \\
Level 3: Urgent & $156(44.4 \%)$ & $130(50.4 \%)$ & \\
Level 4: Less urgent & $55(15.7 \%)$ & $38(14.7 \%)$ & \\
Level 5: Non-urgent & $7(2.0 \%)$ & $10(3.9 \%)$ & 0.16 \\
Mode of arrival, $n$ (\%) & & & \\
Ambulance & $99(28.2 \%)$ & $59(22.9 \%)$ & $196(76.0 \%)$ \\
Personal Means & $247(70.4 \%)$ & $3(1.2 \%)$ & \\
Missing & $1(0.3 \%)$ & $3.7 \pm 5.6$ & 0.98 \\
Symptom duration (days) & $3.7 \pm 6.2$ & $20.1 \pm 16.0$ & $<\mathbf{0 . 0 0 1}$ \\
Length of ED stay (hours) & $25.2 \pm 20.7$ & $1.4 \pm 1.4$ & $<\mathbf{0 . 0 0 1}$ \\
Time to be seen by MD (hours) & $2.8 \pm 2.8$ & $4.6 \pm 5.8$ & 0.86 \\
Time to completion of consults (hours) & $4.7 \pm 7.2$ & $12.4 \pm 8.4$ & $\mathbf{0 . 0 2}$ \\
Time to admission (hours) & $14.6 \pm 11.9$ &
\end{tabular}

Data are presented as $n$ (\% total) or mean \pm standard deviation

ED Emergency Department, $M D$ Medical Doctor
Table 2 Emergency department care characteristics for general surgery patients during the pandemic (March13-May13, 2020) and pre-pandemic (March13-May13, 2019) periods
Among patients with appendicitis and gallstone disease, rates of operative treatment were similar in the pandemic and pre-pandemic periods. 30-day outcomes including ED revisits $(16.8 \%$ [2019] versus $17.4 \%$ [2020], $p=0.84)$, (re) admissions (8.3\% [2019] versus $10.9 \%$ [2020], $p=0.28$ ), and complications (17.1\% [2019] versus $22.1 \%$ [2020], $p=0.12$ ) were not significantly different between the two periods (Table 3 ).

The risk-adjusted comparison of primary and secondary outcomes is described in Table 4. After adjusting for covariates, the rate of ED visits for general surgery diagnoses was $25 \%$ lower during the pandemic period versus the pre-pandemic baseline [adjusted rate ratio $=0.75$, 
Fig. 1 Distribution of general surgery diagnoses in the emergency department during the pandemic (March13-May13, 2020) and pre-pandemic (March13-May13, 2019) periods.*Indicates statistical significance $(p<0.05)$. Bowel obstruction diagnosis consists small and large bowel obstruction regardless of cause. Gallstone diseases are defined as patients with biliary colic, acute cholecystitis, choledocholithiasis and stone cholangitis. Cholangitis category includes all non-stone causes of cholangitis such as post instrumentalization, stent and obstructing mass. Soft tissue infection consists of cellulitis, abscess, and necrotizing fasciitis

Table 3 In-hospital course and 30-day outcomes during the pandemic (March13-May13, 2020) and pre-pandemic (March13-May13, 2019) periods

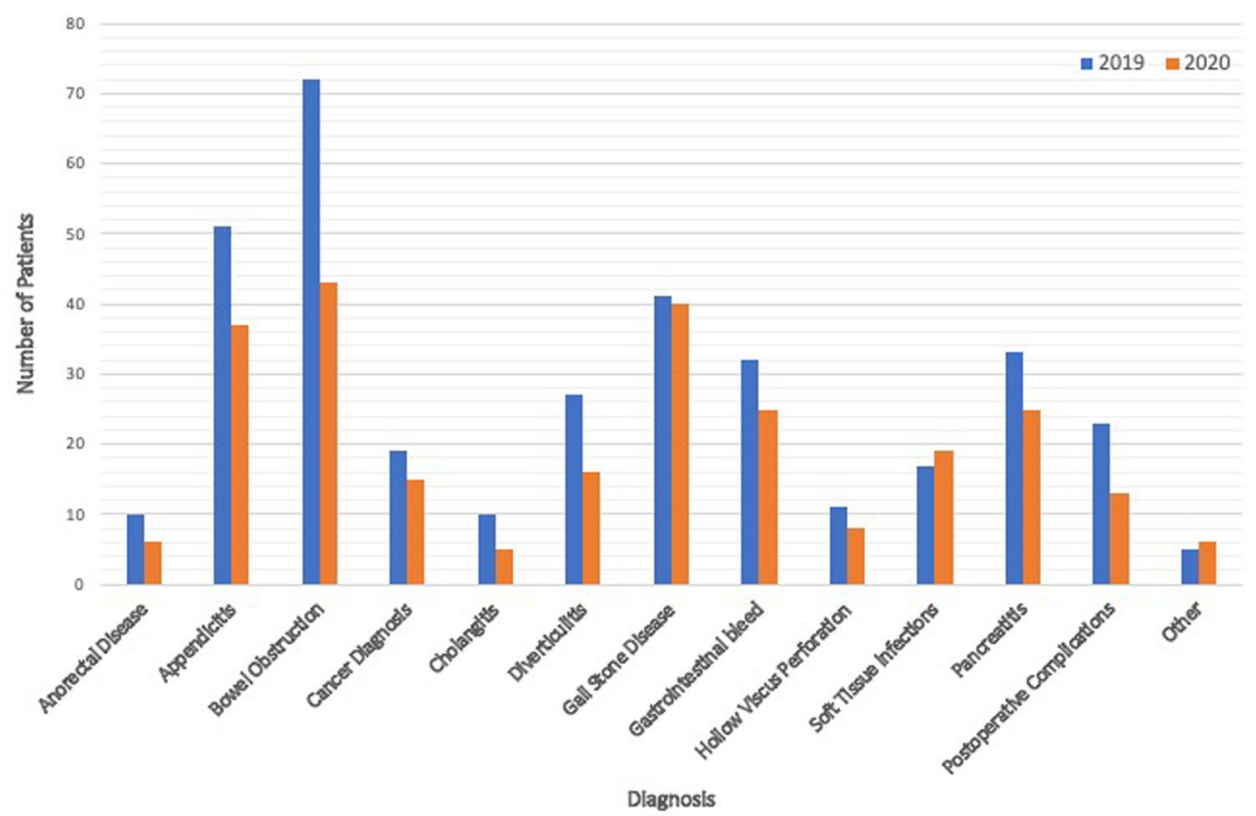

\begin{tabular}{|c|c|c|c|}
\hline & 2019 & 2020 & $p$-value* \\
\hline Hospital admissions, $n(\%)$ & $235(67.0 \%)$ & $179(69.4 \%)$ & $<0.001$ \\
\hline Covid-19 testing (positive/total tested) & NA & $3 / 138$ & NA \\
\hline \multicolumn{4}{|l|}{ Treatment } \\
\hline Interventional radiology, $\mathrm{n}(\%)$ & $28(8.0 \%)$ & $26(10.1 \%)$ & 0.37 \\
\hline Surgery, $n(\%)$ & $115(32.8 \%)$ & $84(32.6 \%)$ & 0.96 \\
\hline Appendicitis treated with surgery, $n$ ( $\%$ of all appendicitis) & $40(78.4 \%)$ & $26(70.3 \%)$ & 0.38 \\
\hline Gallstone disease treated with surgery, $n$ ( $\%$ of all Gallstone disease) & $22(56.4 \%)$ & $19(52.8 \%)$ & 0.57 \\
\hline ICU Admission, $n(\%)$ & $26(7.4 \%)$ & $18(7.0 \%)$ & 0.84 \\
\hline 30-day ED revisit, $n(\%)$ & $59(16.8 \%)$ & $45(17.4 \%)$ & 0.84 \\
\hline 30-day readmission, $n(\%)$ & $29(8.3 \%)$ & $28(10.9 \%)$ & 0.28 \\
\hline 30-day complications, $n(\%)$ & $60(17.1 \%)$ & $57(22.1 \%)$ & 0.12 \\
\hline Intra-abdominal bleeding/infection/sepsis & $13(21.7 \%)$ & $14(24.6 \%)$ & \\
\hline Medical complications & $17(28.3 \%)$ & $12(21.1 \%)$ & \\
\hline Recurrence & $21(35 \%)$ & $16(28.1 \%)$ & \\
\hline Pain & $1(1.7 \%)$ & $10(17.5 \%)$ & \\
\hline Postoperative ileus & $4(6.7 \%)$ & $3(5.3 \%)$ & \\
\hline Wound complications & $4(6.7 \%)$ & $2(3.5 \%)$ & \\
\hline In-hospital death, $n(\%)$ & $8(2.3 \%)$ & $10(3.9 \%)$ & 0.25 \\
\hline Length of hospital stay (days) & $8.7 \pm 14.0$ & $6.3 \pm 9.5$ & $\mathbf{0 . 0 3}$ \\
\hline ICU length of stay (days) & $8.5 \pm 13.4$ & $5.6 \pm 9.8$ & 0.40 \\
\hline
\end{tabular}

Data are presented as $n$ (\% patients presenting to the emergency department) or mean \pm standard deviation ICU Intensive Care Unit, ED Emergency Department $p<0.001]$. Hospital admissions where 23\% lower [adjusted rate ratio $=0.77, \mathrm{p}<0.001$ ]. The risk-adjusted ED length of stay was significantly shorter during the pandemic period (adjusted mean difference $=-5.0 \mathrm{~h}$, $p<0.001)$. The risk-adjusted hospital length of stay was not significantly different between the two periods (adjusted mean difference $=-1.67$ days, $p=0.14$ ). Similarly, risk-adjusted 30-day outcomes (complication, ED revisits and readmission) were not significantly different between the pandemic and pre-pandemic periods (Table 4). 
Table 4 Risk-adjusted comparison of primary and secondary outcomes between the pandemic (March13-May13, 2020) and pre-pandemic (March13-May13, 2019) period

\begin{tabular}{|c|c|c|c|}
\hline & Adjusted rate ratio $^{a}$ & Confidence interval & $p$-value \\
\hline ED visits with general surgery diagnosis & 0.75 & $0.64,0.88$ & $<0.001$ \\
\hline \multirow[t]{2}{*}{ Hospital admissions with general surgery diagnosis } & 0.77 & $0.63,0.94$ & $<0.001$ \\
\hline & Adjusted mean difference ${ }^{b}$ & Confidence interval & $p$-value \\
\hline ED length of stay, hours & -5.0 & $-8.1,-2.0$ & $<0.001$ \\
\hline Hospital length of stay, days & -1.67 & $-3.89,0.54$ & 0.14 \\
\hline \multirow[t]{2}{*}{ Duration of symptoms prior to presentation, days } & 0.06 & $-0.93,1.06$ & 0.90 \\
\hline & Adjusted odds ratio ${ }^{c}$ & Confidence interval & $p$-value \\
\hline 30-day ED revisit & 1.10 & $0.70,1.71$ & 0.66 \\
\hline 30-day readmission & 1.42 & $0.81,2.48$ & 0.22 \\
\hline 30-day complication & 1.46 & $0.97,2.22$ & 0.07 \\
\hline
\end{tabular}

${ }^{a}$ Adjusted rate ratio signifies the rate of ED visits for general surgery diagnosis during the pandemic versus the pre-pandemic baseline after adjusting for covariates

${ }^{\mathrm{b}}$ Adjusted mean difference signifies the difference in length of ED stay during the pandemic versus the pre-pandemic baseline after adjusting for covariates

${ }^{\mathrm{c}}$ Adjusted odds ratio signifies the odds of having a 30-day complication in patients presenting to ED with general surgery conditions during the pandemic period versus the pre-pandemic baseline after adjusting for covariates

All regression analyses were adjusted for sex, age, Charlson Comorbidity Index, psychiatric comorbidities, and income quantiles

ED Emergency department

\section{Discussion}

In this study, we identified a significant decrease in riskadjusted rates of ED utilization by patients with general surgery pathologies during the first wave of the Covid-19 pandemic (25\% reduction in comparison to the pre-pandemic baseline). Among these patients, there was also a significant decrease in hospital admissions (23\% reduction comparted to the pre-pandemic baseline). Our findings are in keeping with a trend of reduced overall ED usage by patients due to the Covid-19 pandemic in Canada and internationally [6, $8,26-28]$. In our study, there were no differences in the acuity of presentations based on the Canadian triage and acuity scale. This highlights that the observed decrease in care-seeking behaviour was not only limited to patient with non-urgent pathologies but extended to more urgent conditions with possible serious consequences [9, 26, 29]. Length of symptoms before ED presentation during the pandemic was comparable to the pre-pandemic period. Rates of ED presentations for more severe conditions such as hollow viscous perforation, or more worrisome visual symptoms such as severe soft tissue infection and gastrointestinal bleeding were also not different between the two time periods. However, during the pandemic, we observed a significant decrease in presentations for conditions that often present with more general symptoms (low-grade fever, abdominal pain, or vomiting) such as pancreatitis, appendicitis, bowel obstruction and diverticulitis. Although there is increasing evidence that some of the latter conditions can be successfully treated with conservative management [30-33], the impact of delayed ED presentation and treatment initiation after symptom onset remains uncertain and increased morbidity cannot be excluded.

In comparison to the pre-pandemic baseline, patients with general surgery conditions presenting to the ED during the pandemic experienced a significantly lower length of ED length of stay, waiting time before assessment by a medical doctor and time to hospital admission. This was likely the result of institutional directives to optimize workflow in ED intake (i.e. faster patient assessment) and discharge (faster disposition decision) [34], gaining momentum from previous initiatives aimed at decreasing ED process times in Canada and the United States [28, 34]. In our institutions, an observed improvement in hospital bed management, communication between admitting services and emergency providers has been instrumental for improving ED care during the pandemic. Diverting simpler cases to primary care clinics and using telehealth has also been of great value. Moving forward, lessons learned during the pandemic may help diverting non-emergent patients from hospitals, enabling a better balance between supply and demand for ED care [34]. Even though the institutional directives to optimize patient flow and disposition planning were directed to both emergency department and hospital wards, length of stay was only significantly decreased in the ED but not in inpatient units. This can be explained by the fact that 
our center has a longstanding enhanced recovery culture at postoperative wards; [35] therefore, it is possible that flow of patients through these units was already optimized before the pandemic.

Although, during the pandemic, we observed an absolute decrease in the number of emergency operations owing to the decreased rate of ED presentation, the proportion of general surgery conditions treated with an operation or interventional radiology remained stable compared to the prepandemic baseline. Similar trends were seen when patients presenting with appendicitis and gallstone disease were analysed separately. Furthermore, we found that risk-adjusted 30-day outcomes (complications, ED revisits and (re)admissions) were not significantly different in patients presenting to the ED during the pandemic versus the pre-pandemic period. Previous literature has described an increase in complications rates among patients with cardiac conditions and patients undergoing emergency general surgery during the pandemic [29, 36]. In our cohort, proficient patient flow management, coordination and communication between our network hospitals potentially contributed to the uphold of standards of care and positive patient outcomes. For example, whenever a patient in need for surgery presented to a network hospital experiencing limited OR availability due to Covid-related resource restrictions, they were transferred to another hospital for timely operation.

Understanding the reasons for avoidance of ED is crucial for devising future interventions to avoid unnecessary health seeking delays during and beyond the pandemic [10, 29]. This assessment, which was not within the scope of our work, has been addressed in recent literature. Wong et al. used qualitative patient interviews to identify reasons for ED avoidance during the pandemic and introduce actionable solutions to encourage a more educated ED utilization among patients [29]. The most common identified reasons for ED avoidance were as follows: (a) perception of hospitals as infectious reservoirs, (b) lack of awareness about hospitals' risk mitigation efforts, and (c) disconnect between vulnerable population and their support network who assist with healthcare visits [29]. Proposed solutions included physical division of the ED to separate patients receiving care for respiratory illness from other patients, and transparent communication regarding risk mitigation strategies used in the hospital [29]. The use of such strategies may further encourage patients with general surgery conditions to seek ED care in a timely manner.

To our knowledge, this study is the first to describe the patterns of ED utilization by patients with general surgery conditions during the Covid-19 pandemic. Our findings add to previous literature assessing rates and outcomes of emergency general surgery procedures and general surgery admissions during the pandemic [37]. A strength of our study is that we used rigorous statistical methodology, adjusting our analysis for multiple confounders including medical and psychiatric comorbidities, and socioeconomical status. Our study was conducted in a large academic health network with a catchment area of 1.9 million persons encompassing $63 \%$ of the provincial landmass [38]. The network includes quaternary, tertiary, and community hospitals and was significantly involved and impacted by the Covid-19 pandemic. Our results should be interpreted with some limitations in mind. Given the nature of our study, we did not conduct a formal sample size calculation; therefore, some of our non-statistically significant results may be due to type II error. Data regarding patient outcomes (i.e. complications, ED revisits, (re)admissions) were obtained via review of institutions electronic medical records. Therefore, visits and complications detected in other hospitals may not have been captured. However, as per provincial regulations, patients presenting to another hospital would be repatriated to their 'home' hospital after initial work-up (i.e. if a patient had a cholecystectomy at the MUHC and presents with bile leak to another hospital in Montreal, they will be transferred back to the MUHC for admission and management). It is important to note that our findings only apply to general surgery patients who presented to our health network's ED during the first wave of the pandemic and not to patients who did not seek ED care. We were not able to find other studies in the literature addressing this question, this could be due to the difficulty of reliably identifying this population. Studies with longer term follow-up would be better for characterizing rates and patterns of morbidity in patients with delayed ED presentation.

In conclusion, our study supports that there was a significant decrease in rates of ED utilization and hospital admissions due to general surgery conditions during the first wave of the Covid-19 pandemic. This finding corroborates trends seen in ED utilization by patients with other medical conditions [6, 28]. Reduced ED utilization was observed across a wide range of disease acuities and extended beyond nonurgent conditions. We were able to uphold our institutional standard of care for general surgery conditions, as demonstrated by stable rates of operative management, complications and healthcare reutilization. This was made possible with proficient patient flow management and effective coordination between our network hospitals.

Supplementary Information The online version contains supplementary material available at https://doi.org/10.1007/s00464-021-08956-3.

Funding Fonds de la recherche en Sante du Quebec (FRSQ).

\section{Declarations}

Disclosures Saba Balvardi, Josie Cipolla, Nawar Touma, Tharaniya Vallipuram, Natasha Baron, Reginold Sivarajan, Pepa Kaneva, Sebas- 
tian Demyttenaere, Marylise Boutros, Lawrence Lee, Liane S Feldman and Julio F Fiore Jr have no conflict of interest to declare.

\section{References}

1. Li JY, You Z, Wang Q, Zhou ZJ, Qiu Y, Luo R et al (2020) The epidemic of 2019-novel-coronavirus (2019-nCoV) pneumonia and insights for emerging infectious diseases in the future. Microbes Infect 22(2):80-85

2. Québec Gd. Data on COVID-19 in Québec. Gouvernement du Québec; 2021.

3. Organization WH 2020;Pages. Accessed at World Health Organization at https://www.who.int/news-room/q-a-detail/herd-immun ity-lockdowns-and-covid-19 on July 30, 20212021.

4. Authier P. Quebec will move medical staff to Montreal's coronavirus battlefront. Montreal Gazette. 2020; April 20, 2020.

5. Fung THM, Kuet ML, Patel MK, Puri P (2021) Addressing COVID-19 fear to improve clinic attendance for patients with wet age-related macular degeneration. Acta Ophthalmol. 99(2):e285

6. De Filippo O, D’Ascenzo F, Angelini F, Bocchino PP, Conrotto F, Saglietto A et al (2020) Reduced rate of hospital admissions for ACS during Covid-19 outbreak in Northern Italy. N Engl J Med 383(1):88-89

7. Hartnett KPK-PA, DeVies J et al (2020) Impact of the COVID-19 pandemic on emergency department visits-United States, January 1, 2019-May 30, 2020. Centers Dis Control Prev 23:699-704

8. Metzler B, Siostrzonek P, Binder RK, Bauer A, Reinstadler SJ (2020) Decline of acute coronary syndrome admissions in Austria since the outbreak of COVID-19: the pandemic response causes cardiac collateral damage. Eur Heart J 41(19):1852-1853

9. Guo H, Zhou Y, Liu X, Tan J (2020) The impact of the COVID-19 epidemic on the utilization of emergency dental services. J Dent Sci 15(4):564-567

10. Statistica INd. L'andamento dei decessi del 2020. Dati anticipatori sulla base del sistema ANPR. Italy: Istituto Nazionale di Statistica 2020.

11. Lyu HG, Najjar P, Havens JM (2018) Past, present, and future of emergency general surgery in the USA. Acute Med Surg 5(2):119-122

12. Havens JM, Neiman PU, Campbell BL, Croce MA, Spain DA, Napolitano LM (2019) The future of emergency general surgery. Ann Surg 270(2):221-222

13. Cuschieri S (2019) The STROBE guidelines. Saudi J Anaesth 13(Suppl 1):S31-S34

14. Utter GH, Miller PR, Mowery NT, Tominaga GT, Gunter O, Osler TM et al (2015) ICD-9-CM and ICD-10-CM mapping of the AAST Emergency General Surgery disease severity grading systems: Conceptual approach, limitations, and recommendations for the future. J Trauma Acute Care Surg 78(5):1059-1065

15. Vasavada B, Patel H. Non surgical procedure related postoperative Complications independently predicts perioperative mortality, in gastrointestinal and $\mathrm{Hpb}$ surgeries.-A retrospective Analysis of prospectively maintained data. medRxiv. 2020.

16. Mirhaghi A, Heydari A, Mazlom R, Ebrahimi M (2015) The reliability of the Canadian triage and acuity scale: meta-analysis. N Am J Med Sci 7(7):299-305

17. Austin SR, Wong YN, Uzzo RG, Beck JR, Egleston BL (2015) Why summary comorbidity measures such as the Charlson comorbidity index and Elixhauser score work. Med Care 53(9):e65-72

18. ICD-9-CM. International Classification of Diseases, 9th revision, Clinical Modification. 3d edition, volumes 1, 2 and 3. Official authorized addendum effective October 1, 1990--HCFA. J Am Med Rec Assoc 1990;61(8): 1-35.
19. Birkmeyer JD, Finks JF, O'Reilly A, Oerline M, Carlin AM, Nunn AR et al (2013) Surgical skill and complication rates after bariatric surgery. N Engl J Med 369(15):1434-1442

20. Brajcich BC, Stulberg JJ, Palis BE, Chung JW, Huang R, Nelson $\mathrm{H}$ et al (2021) Association between surgical technical skill and long-term survival for colon cancer. JAMA Oncol 7(1):127-129

21. Barut I, Tarhan OR, Cerci C, Karaguzel N, Akdeniz Y, Bulbul M (2005) Prognostic factors of peptic ulcer perforation. Saudi Med J 26(8):1255-1259

22. Scholz AF, Oldroyd C, McCarthy K, Quinn TJ, Hewitt J (2016) Systematic review and meta-analysis of risk factors for postoperative delirium among older patients undergoing gastrointestinal surgery. Br J Surg 103(2):e21-e28

23. Abrams TE, Vaughan-Sarrazin M, Rosenthal GE (2010) Influence of psychiatric comorbidity on surgical mortality. Arch Surg 145(10):947-953

24. Ultee KHJ, Tjeertes EKM, Bastos Goncalves F, Rouwet EV, Hoofwijk AGM, Stolker RJ et al (2018) The relation between household income and surgical outcome in the Dutch setting of equal access to and provision of healthcare. PLoS One 13(1):e0191464

25. Jakobsen JC, Gluud C, Wetterslev J, Winkel P (2017) When and how should multiple imputation be used for handling missing data in randomised clinical trials-a practical guide with flowcharts. BMC Med Res Methodol 17(1):1-10

26. Podda M, Cillara N, Di Saverio S, Lai A, Feroci F, Luridiana G et al (2017) Antibiotics-first strategy for uncomplicated acute appendicitis in adults is associated with increased rates of peritonitis at surgery. A systematic review with meta-analysis of randomized controlled trials comparing appendectomy and nonoperative management with antibiotics. Surgeon 15(5):303-14

27. Tam CF, Cheung KS, Lam S, Wong A, Yung A, Sze M et al (2020) Impact of Coronavirus Disease 2019 (COVID-19) outbreak on ST-segment-elevation myocardial infarction care in Hong Kong, China. Circ Cardiovasc Qual Outcomes. 13(4): 006631

28. Information CIfH. How COVID-19 affected emergency departments. Canadian Institute for Health Information 2021.

29. WongLaura E, HawkinsJessica E, MurrellKaren L. Where are all the patients? Addressing Covid-19 fear to encourage sick patients to seek emergency care. NEJM Catalyst Innovations in Care Delivery. 2020.

30. Huston JM, Zuckerbraun BS, Moore LJ, Sanders JM, Duane TM (2018) Antibiotics versus no antibiotics for the treatment of acute uncomplicated diverticulitis: review of the evidence and future directions. Surg Infect (Larchmt) 19(7):648-654

31. Collaborative C, Flum DR, Davidson GH, Monsell SE, Shapiro NI, Odom SR et al (2020) A randomized trial comparing antibiotics with appendectomy for appendicitis. N Engl J Med 383(20):1907-1919

32. Jackson P, Raiji MT (2011) Evaluation and mangement of intestinal obstruction. Am Fam Physician 83(2):159-165

33. Garfinkle R, Salama E, Amar-Zifkin A, Morin N, Ghitulescu G, Faria J et al (2021) Observational versus antibiotic therapy for acute uncomplicated diverticulitis: A non-inferiority metaanalysis based on a Delphi consensus. Surgery. https://doi.org/ 10.1016/j.surg.2021.07.012

34. Woodruff A, Frakt AB. COVID-19 pandemic leads to decrease in emergency department wait times. JAMA Health Forum, 2020. American Medical Association: e201172-e.

35. 2012;Pages. Accessed at McGill University Health Centre at https://muhc.ca/health-info/article/surgery-recovery-program on Nov 28, 20212021.

36. Perea Del Pozo E, Aparicio-Sanchez D, Hinojosa Ramirez F, Pareja Ciuro F, Duran Munoz-Cruzado V, Sanchez Arteaga A et al (2020) A prospective cohort study of the impact of covid19 world 
pandemic on the management of emergency surgical pathology. Br J Surg 107(11):e463-e464

37. Hessheimer AJ, Trapero-Bertran M, Borin A, Butori E, Curell A, Espinoza AS et al (2021) Resource utilization and outcomes in emergency general surgery during the COVID19 pandemic: an observational cost analysis. PLoS One. 16(6):e0252919

38. Centre MUH 2021;Pages. Accessed at McGill University Health Centre at https://muhc.ca/health-professional-information/ruisinformation on June 29, 20212021.
Publisher's Note Springer Nature remains neutral with regard to jurisdictional claims in published maps and institutional affiliations. 